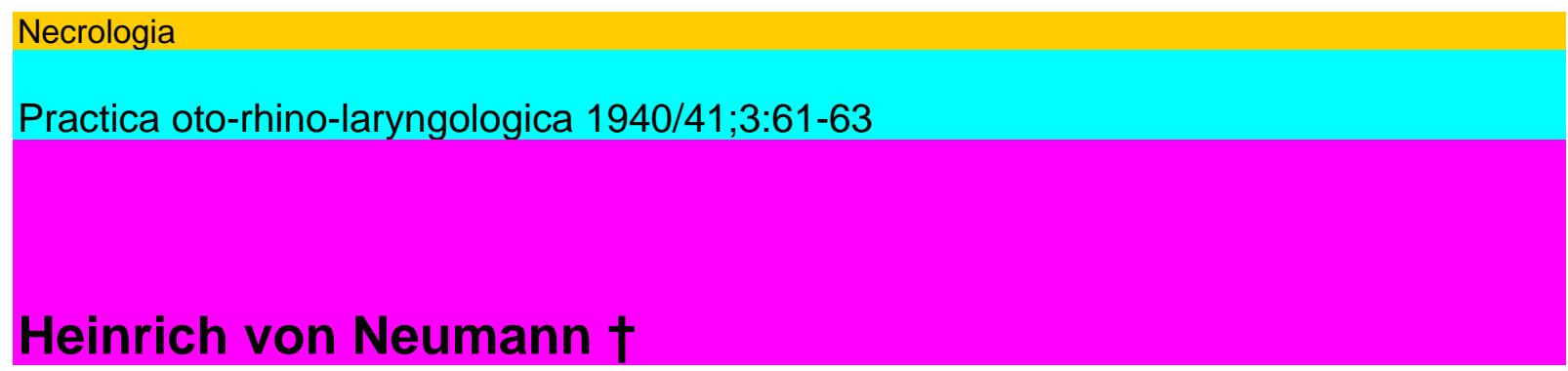

\title{
K. Eisinger
}

\section{Edinbrgh}

Heinrich v. Neumann wurde am 10. Juli 1873 in Miskolcz (Ungarn) geboren, besuchte dortselbst das Gymnasium und in-skribierte dann an der Wiener Universität erst Jus, dann nach zwei Semestern Medizin. Im Beginne seines Studiums war er Demonstrator am Pflanzenphysiologischen Institut. Bald nach Beginn der klinischen Semester trat er in die Ohrenklinik ein und arbeitete auf Rat Polítzers gleichzeitig am pathologisch-anatomischen und bakteriologischen Institut bei Prof. Weíchsel-baum. Nach seiner Promotion war er Operateur und von 1903 an Assistent bei Adam Pulitzer. Als Lieblingsschüler dieses großen Mannes hatte er Gelegenheit, die klinische und wissenschaftliche Ausbildung zu genießen, die die Grundlage seines späteren Wir-kens und Schaffens war.

Im Jahre 1907 habilitierte sich v. Neumann an der Wiener Universität als Privatdozent mit einer Monographic über den otogenen Kleinhirnabszeß. Diese Monographie, die vor 33 Jahren 62

\section{Heinrich von Neumann $\dagger$}

geschrieben wurde, ist noch heute die Basis fur jeden, der über dieses Gebiet arbeitet. Er hat darin unter anderem als erster auf die Verwertung des Nystagmus als Differentialdiagnostikum zwischen Labyrinthitis und Kleinhirnabszeß hingewiesen. Sein Name war zu dieser Zeit schon bekannt durch die Neumannsche Labyrinthoper ation.

Im selben Jahre erhielt er als erster Vertreter der Wiener Schule eine Einladung, an amerikanischen Universitäten Vorträge zu halten. Die Technik der Labyrinthoperation, die Klinik der Streptococcus mucosus otitis, seine Methode der Operation des Warzenfortsatzes in Lokalanästhesie und die Pathologie und Therapie otogener endokranieller Komplikationen waren seine hauptsächlichen Arbeitsgebiete. Die Vorträge Neumanns wurden mit Begeisterung aufgenommen und hatten eine bedeutende Zu-nahme des Zustromes ausländischer Aerzte nach Wien zur Folge. Im Jahre 1908, mit dem Abgange Polítzers, verließ Neumann die Klinik und arbeitete bis 1914 an seiner Abteilung. In diese Zeit fallen seine Arbeiten über Bulbusthrombose und die Bedeu-tung der akuten Exazerbation der chronischen Ohren- und Naseneiterungen. Im Jahre 1913 wurde ihm in Anerkennung seiner wissenschaftlichen Leistungen der Titel Universitätspro-fessor verliehen. Nach dem Kriege erhielt Neumann die Berufung als Vorstand der Wiener Ohren-Nasen-Kehlkopf-Klinik und leitete die Klinik bis März 1938. Nach wie vor war sein Lieblingsgebiet die Klinik der otogenen Komplikationen. Die Histopathologie der akuten Otitis, die konservative Radikaloperation, Technik der Mastoidektomie, Pathologie des Groß- und Kleinhirnabszesses, die operative Behandlung der otogenen Meningitis, die Bedeutung der frühzeitigen Lumbalpunktion und die Indikation zur Labyrinthoperation stellen nur einen Teil der Gebiete dar, über die er arbeitete und mit denen sein Name dauernd verknüpft ist.

Es ist unnötig, in einem Nachrufe, der für Fachkollegen bestimmt ist, die alle v. Neumann kannten und arbeiten gesehen hatten, seine Leistungen als Fachmann noch einmal aufzuzählen. 
Dafür ist er und ist alles, was er geschaffen hat, noch zu frisch in unserer Erinnerung. Beweise der Anerkennung seiner Tätig-keit sind die zahlreichen Ernennungen zum Ehrenmitglied der hervorragendsten Fachgesellschaften der ganzen Welt. Es gab keinen Kongreß, auf dem seine Anwesenheit nicht mit Freude begrüßt wurde, und seine Vorträge und Diskussionen bildeten Heinrich von Neumann $\dagger$

63

den Mittelpunkt des Interesses. Wer konnte, kani nach Wien, urn Neumann arbeiten und operieren zu sehen, und zahllose Fach-ärzte aus alien Erdteilen kamen, um an seiner Klinik weitere Ausbildung zu genießen. Seine Vorlesung für Studenten war die bestbesuchte der Wiener medizinischen Fakultät, und seine letzte Vorlesung hielt er sechs Stunden vor dem Einmarsch der Deut-schen in einem bis auf den letzten Platz gefüllten Hörsaale.

Es gibt kauni ein Land, aus dem nicht Patienten kamen, um bei diesem großen Arzt Hilfe zu suchen. Könige und Fürsten, führende Politiker, Finanzmagnaten, Menschen, die das Schicksal von Völkern in der Hand hatten, kamen zu ihm auf Grund seines Namens und verehrten ihn wegen seiner Persönlichkeit. Er konnte helfen, wo andere versagten, und dies nicht nur durch seine überragenden Fachkenntnisse, sondern durch sein Wesen und durch niewiederkehrende Art, Patienten zu beeinflussen. Seine Liebe zu den klinischen Patienten, insbesondere zu Kindern, war der Grund der Liebe und Verehrung, die er bei den Spitalpatienten genoß.

Was v. Neumann seinen Assistenten bedeutete und was jeder von ihnen ihm zu verdanken hatte, das können nur die schätzen, die das Glück hatten, seine nächsten Mitarbeiter zu sein. Jede Visite im Krankensaal, die Besprechung jedes Falles, die Stellung und Lösung von Problemen, die unvergleichliche Art, sein klini-sches Wissen und seine Erfahrung uns zu übermitteln, ist jedem unvergeßlich. Als Arzt und Wissenschaftler mag v. Neumann vielleicht ersetzlich sein, aber unersetzlich ist er als Lehrer und als Vermittler der klinischen Ohrenheilkunde, die unter Polítzer und ihm einen Aufschwung nahm, wie er in wenigen medizinischen Fächern aufzuweisen ist. Als Wissenschaftler hatte er vielleicht Gegner aber nie Feinde. Als Mensch und Arzt hatte er nur Freunde und Verehrer. Alle die ihn kannten und denen er durch seine ärztliche Kunst oder durch seine menschliche Güte geholfen hat werden v. Neumann nie vergessen. Seine Leistungen werden uns alle überleben.

K. Eisinger

Edinburgh. 